\title{
Comparative Assessment of Gingival Overgrowth: A Clinicopathologic Study
}

\author{
Shruti Srinivasan ${ }^{1}$, NS Priya ${ }^{2}$, Kavita Rao ${ }^{3}$, HS Umadevi ${ }^{4}$, T Smitha ${ }^{5}$, HS Sheethal ${ }^{6}$
}

\begin{abstract}
Introduction: Gingival overgrowth $(\mathrm{GO})$ is the increase in the size of the gingiva due to various causes. The knowledge about their diverse clinical and histopathological features helps us in their diagnosis, treatment, and management.

Aim and objective: To understand the clinical and the histopathological spectrum of inflammatory gingival overgrowth (IGO), drug-induced gingival overgrowth (DIGO), and hereditary gingival overgrowth (HGO).

Settings and design: Ninety histopathologically diagnosed cases of IGO, DIGO, and HGO were studied.

Materials and methods: 5 micron sections were stained with $\mathrm{H} \& \mathrm{E}$. The clinical and the histopathological variables of epithelium and connective tissue were compared.

Statistical analysis used: Chi-squared test was done to compare the variables among the groups.

Results: The parameters, namely, age, site, color, consistency, shape, epithelium, rete ridges, inflammation, inflammatory cell predominance, vascularity, fibrosis, and hyalinization showed significant variation between the study groups.

Conclusions: In this study, IGO, DIGO, and HGO showed a female predominance and most cases of IGO and HGO occurred in the 3rd-4th decade whereas DIGO in the 5th-6th decade. Clinically, IGO appeared erythematous and soft in consistency compared to DIGO and HGO, which appeared pink clinically and firm in consistency and all the three study groups showed a site predilection of the maxillary anterior region. Histopathologically IGO showed more inflammatory components than DIGO and HGO which showed more fibrotic connective tissues than IGO. Keywords: Drug-induced gingival overgrowth, hereditary gingival overgrowth, inflammatory gingival overgrowth.

Journal of Health Sciences \& Research (2020): 10.5005/jp-journals-10042-1093
\end{abstract}

\section{INTRODUCTION}

Gingival tissue exhibits a unique behavior, being in a permanent state of injury and repair, which further involves repetitive production of chemotactic factors, inflammatory cell recruitment, and tissue remodeling. ${ }^{1}$ Increase in the size of the gingiva is a common feature of gingival disease. Accepted current terminology for this condition is called gingival enlargement or gingival overgrowth (GO). These are strictly clinical-descriptive terms. $^{2}$

Gingival overgrowth is an umbrella term which encompasses gingival enlargement due to inflammation, drug influence, and hereditary factors. Studies have demonstrated morphological and histopathological diversities between the GO caused by various etiologies.

Exuberant response of gingival tissue to local irritants is the most common cause of GO. Increase in the size of the gingival tissue can also be seen as an after-effect due to certain systemic drugs known as drug-induced gingival overgrowth (DIGO). Variation in the levels of estrogen and progesterone in pregnant women and during puberty has effects on gingiva and may present as overgrowth. It has also been attributed to genes and certain chromosomal abnormalities and hence maybe hereditary, part of the syndromes appears as solitary pathologies which are put under hereditary gingival overgrowth (HGO).

Despite the various etiologies causing a similar consequence which is seen as an overgrowth, there are distinct clinical and histopathological differences amongst them.
1Department of Oral Pathology, Oxford Dental College, Bengaluru, Karnataka, India

${ }^{2-6}$ Department of Oral and Maxillofacial Pathology, VS Dental College and Hospital, Bengaluru, Karnataka, India

Corresponding Author: Shruti Srinivasan, Department of Oral pathology, VS Dental College and Hospital, Bengaluru, Karnataka, India, Phone: +91 9900096081, e-mail: shrutiananthu@gmail.com

How to cite this article: Srinivasan S, Priya NS, Rao K, et al. Comparative Assessment of Gingival Overgrowth: A Clinicopathologic Study. J Health Sci Res 2020;11(2):37-41.

Source of support: Nil

Conflict of interest: None

Molecular studies implicate the concept of epithelialmesenchymal transition (EMT) and modulation of apoptosis in the gingival tissues which contribute to the pathogenesis of gingival fibrosis.

The present study was undertaken to assess the clinical and the histopathological variables of inflammatory, drug-induced, and hereditary gingival overgrowths.

\section{Materials and Methods}

Ninety histopathologically diagnosed cases of inflammatory, druginduced, and hereditary gingival overgrowths (50 inflammatory, 23 drug-induced, and 17 hereditary) were retrieved from the achieves of our department (Table 1). 
Pyogenic granuloma in pregnant women, gingival overgrowth due to systemic diseases, and neoplastic gingival overgrowths were excluded. Five-micron sections were taken and stained with $\mathrm{H} \& \mathrm{E}$. The clinical and the histopathological variables of epithelium and connective tissue were compared. Each section was studied using binocular light microscope after staining with routine $\mathrm{H}$ \& E stain.

\section{Statistical Analysis}

To compare the parameters and determine the significance between the study groups, chi-squared test was done. Proportions were compared using chi-squared $\left(K^{2}\right)$ test of significance. In all the above tests, " $p$ " value of less than 0.05 was accepted as indicating statistical significance.

\section{Results}

A total of 90 cases comprising of 50 cases of IGO, 23 cases of DIGO, and 17 cases of HGO were evaluated for clinical and histopathological variables.

Table 1: Study sample

\begin{tabular}{ll}
\hline Type of sample & Number of cases \\
\hline IGO & 50 \\
DIGO & 23 \\
HGO & 17 \\
Total cases studied & 90 \\
\hline
\end{tabular}

No relevant family history was recorded in the cases taken for study.

The demographic data (age and sex) and the clinical data with regard to site, size, color, consistency, contour, and shape of gingival overgrowths were recorded. Clinical parameters namely, age, color, consistency, and shape showed a highly significant $(p<0.01)$ variation, whereas parameters namely, sex and site showed a significant $(p<0.05)$ variation amongst the three groups and sex distribution did not show a significant variation amongst the three groups (Table 2). The cases in the study showed a similar site predilection with IGO presenting as erythematous, round to ovoid lesions whereas DIGO and HGO presenting as firm, diffuse overgrowths (Figs 1 to 3 ).

Histopathologically, thickness of epithelium (normal/atrophic/ hyperplastic), rete ridge pattern (bulbous, thin, and narrow, sharp/ pointed), type of connective tissue (fibrous, mixed, loose, and edematous), presence of inflammation, predominant inflammatory cells, vascularity, fibrosis, and hyalinization were evaluated. Histopathological parameters namely, inflammation, predominant inflammatory cells, fibrosis, and hyalinization showed a highly significant $(p<0.01)$ variation and parameters namely, epithelium, rete ridges, and vascularity showed a significant $(p<0.05)$ variation amongst the three groups. Type of connective tissue showed no significant variation amongst the three groups (Table 3).

Histopathologically, the cases in the study showed hyperplastic epithelium with irregular rete ridges with predominant cases having a fibrous stroma. IGO showed moderate-to-severe inflammation, whereas DIGO and HGO showed mild inflammation

Table 2: Comparison of clinical parameters between the groups

\begin{tabular}{lllll}
\hline Clinical parameters & IGO & DIGO & HGO & ' $p^{\prime}$ value \\
\hline Age & 3rd-4th decade & 5th-6th decade & 3rd-4th decade & $<0.001$ \\
Sex & $\mathrm{F}>\mathrm{M}$ & $\mathrm{F}>\mathrm{M}$ & $\mathrm{F}>\mathrm{M}$ & 0.230 \\
Site & Maxillary anterior region & Maxillary posterior region & Maxillary anterior region & 0.003 \\
Color & Erythematous & Pink & Pink & $<0.001$ \\
Consistency & Soft & Firm & Firm & $<0.001$ \\
Contour & Irregular & Irregular & Irregular & - \\
Shape & Round to ovoid & Diffuse & Diffuse & $<0.001$ \\
\hline
\end{tabular}

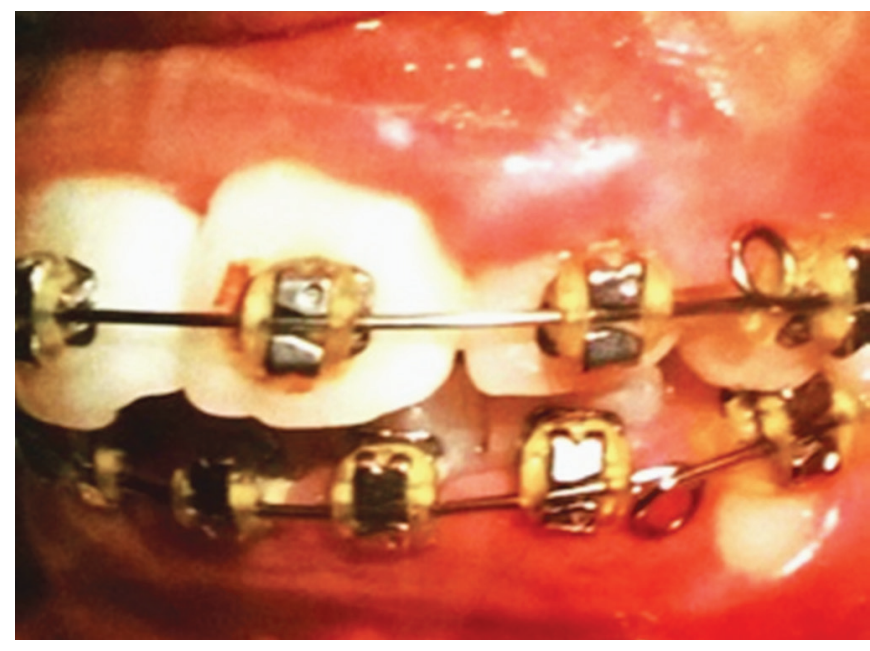

Fig. 1: Clinical picture of IGO affecting the labial mucosa w.r.t central and lateral incisor

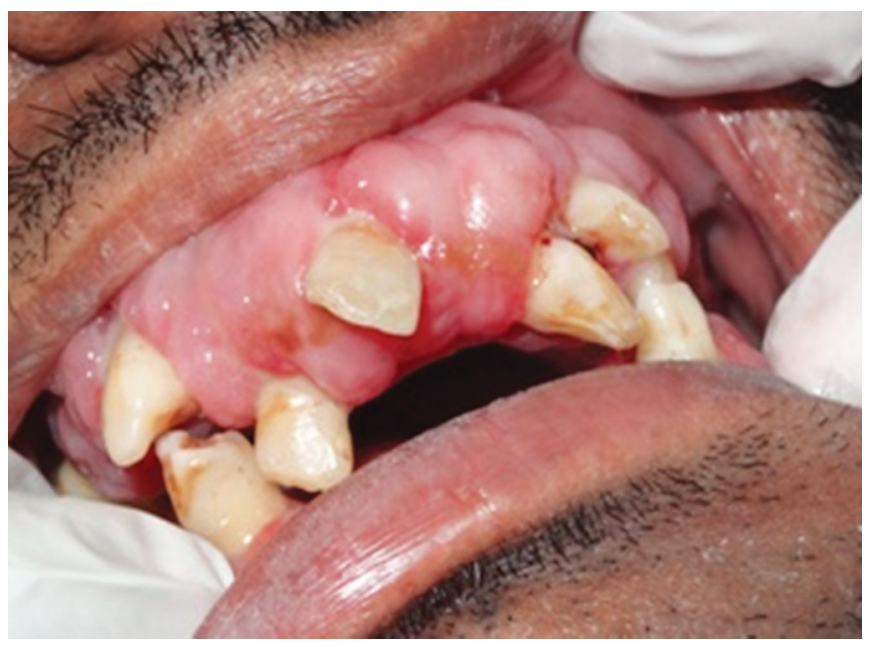

Fig. 2: Clinical picture of DIGO affecting the maxillary arch causing displacement of teeth 
with the predominant inflammatory cells being lymphocytes and plasma cells. Connective tissue of HGO was extensively fibrotic as compared to IGO and DIGO. All the cases of DIGO and HGO showed hyalinization while it was absent in most of the IGO (Figs 2 to 6).

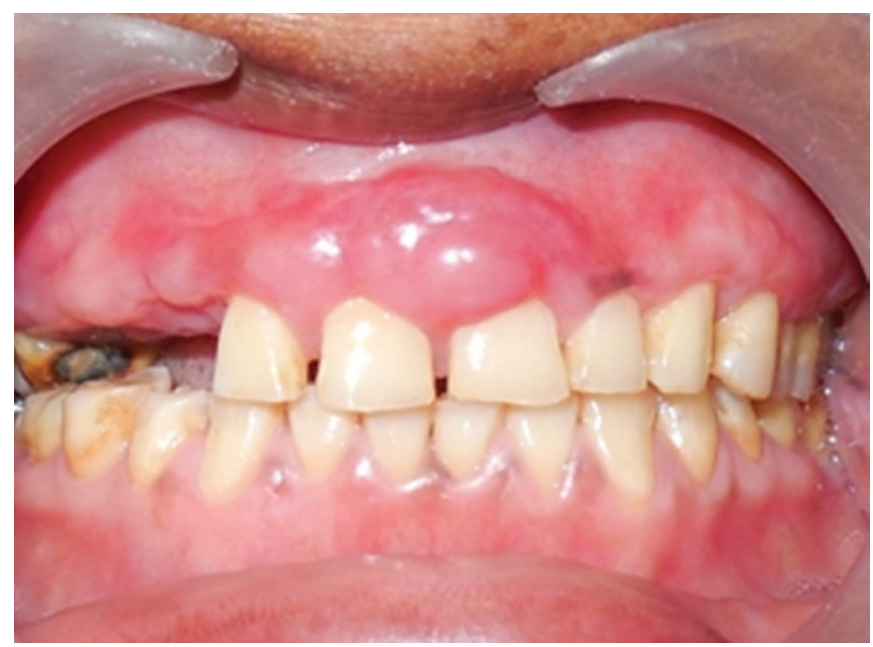

Fig. 3: Clinical picture of localized HGO affecting the maxillary anterior region
A few cases of DIGO showed basal cell budding (Fig. 7) and mast cell distribution.

\section{Discussion}

The term "gingival overgrowth" or "gingival enlargement" must be preferred for all gingival lesions previously termed gingival hypertrophy or gingival hyperplasia due to the fact that gingival enlargement means either accumulation of extracellular matrix or cell proliferation in the same time. ${ }^{1}$

In the present study, 90 cases of histopathologically diagnosed cases of GO which included 50 cases of IGO, 23 cases of DIGO, and 17 cases of HGO were evaluated and compared for clinical and histopathological parameters.

Inflammatory process is the leading cause of gingival overgrowths exhibiting a characteristic clinical presentation and morphologic features. The word "epulis" is derived from Greek "epi" and "elon"; meaning "on the gingiva". 3

Reactive lesions are clinically and histologically non-neoplastic nodular swellings that develop in response to chronic and recurrent tissue injury which stimulates an exuberant or excessive tissue response. ${ }^{4}$ The gingiva is an important area affected by reactive lesions, primarily triggered by chronic inflammation in response to microorganisms in the dental plaque. ${ }^{5} \mathrm{~A}$ fractured tooth, overhanging restorations, ill-fitting prosthesis, orthodontic brackets, etc., can also trigger gingival response which clinically

Table 3: Comparison of histopathological parameters between the groups

\begin{tabular}{|c|c|c|c|c|}
\hline Histopathological parameters & IGO & DIGO & $\mathrm{HGO}$ & 'p'value \\
\hline Epithelium & Hyperplastic & Hyperplastic & Hyperplastic & 0.029 \\
\hline Rete ridges & Irregular & Irregular & Irregular & 0.051 \\
\hline Type of connective tissue & Fibrous & Fibrous & Fibrous & $<0.001$ \\
\hline Inflammation & Moderate-severe & Mild & Mild & $<0.001$ \\
\hline Predominant inflammatory cells & $\begin{array}{l}\text { Lymphocytes and } \\
\text { plasma cells }\end{array}$ & $\begin{array}{l}\text { Lymphocytes and } \\
\text { plasma cells }\end{array}$ & $\begin{array}{l}\text { Lymphocytes and } \\
\text { plasma cells }\end{array}$ & 0.032 \\
\hline Vascularity & Moderate-extensive & Mild & Mild & 0.086 \\
\hline Fibrosis & Mild & Moderate & Extensive & $<0.001$ \\
\hline Hyalinization & Absent & Present & Present & $<0.001$ \\
\hline
\end{tabular}

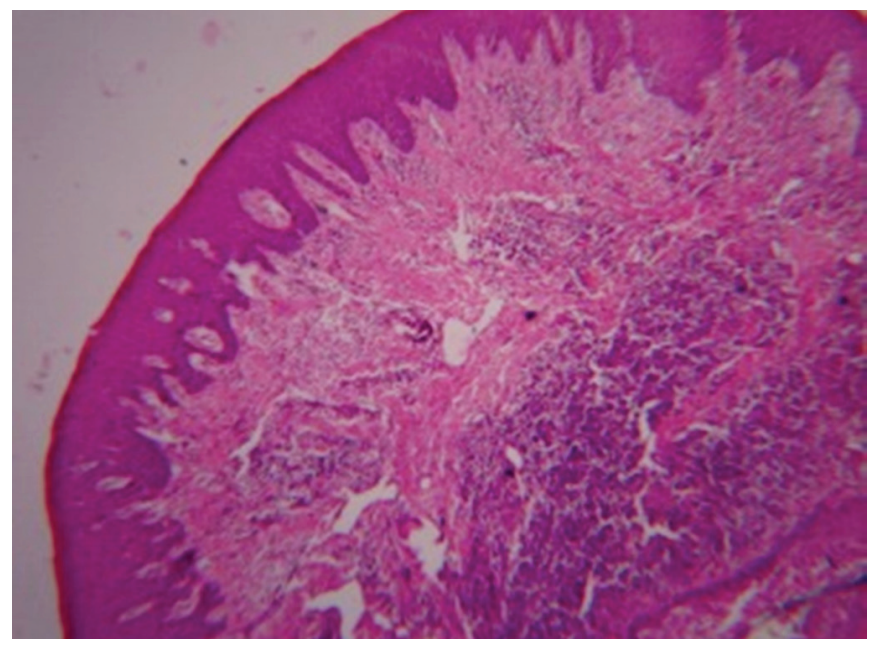

Fig. 4: Histopathological picture of IGO showing hyperplastic epithelium and connective tissue with inflammatory component

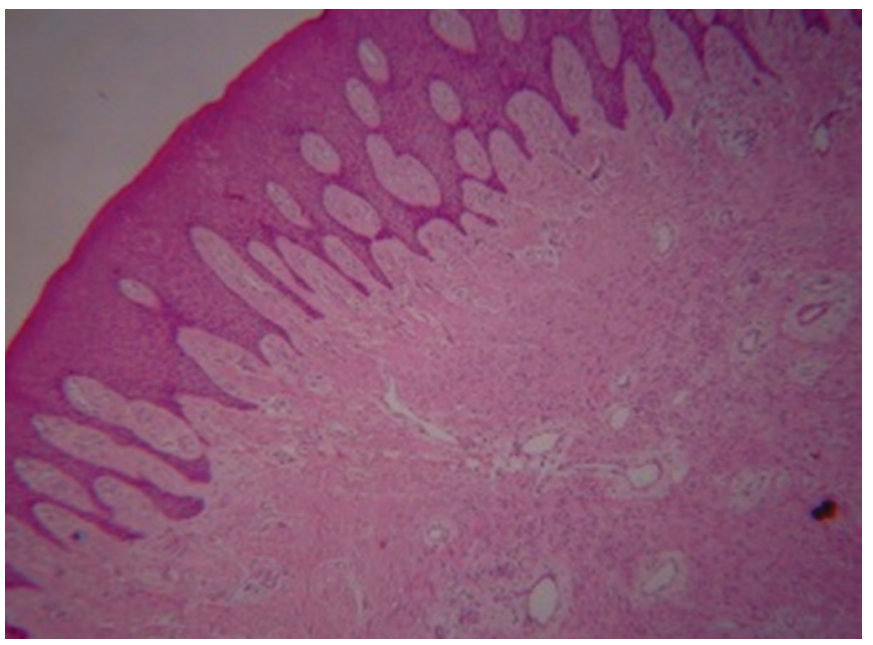

Fig. 5: Histopathological picture of DIGO showing hyperlastic epithelium and fibrous connective tissue with mild inflammatory component 


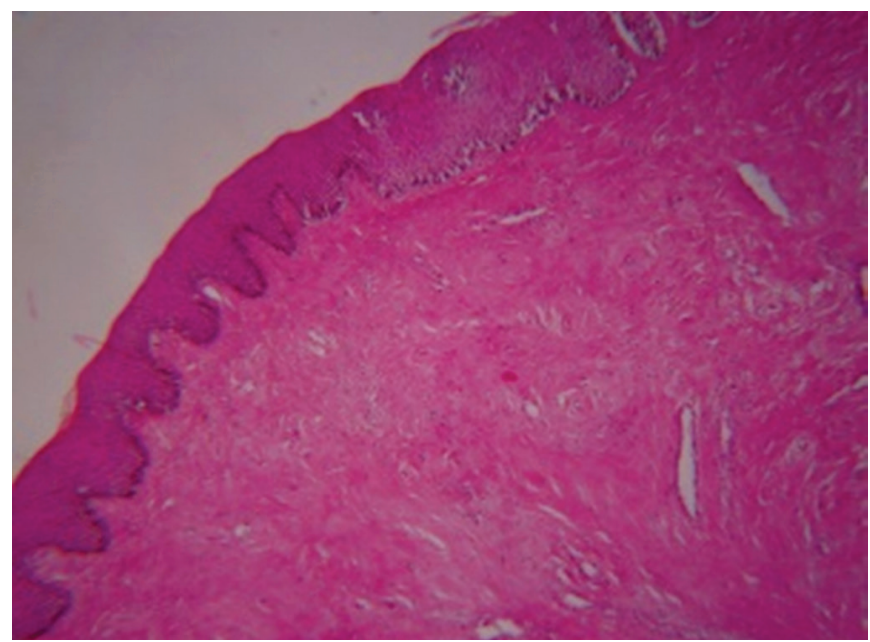

Fig. 6: Histopathological picture of HGO showing hyperplastic epithelium and hyalinized connective tissue

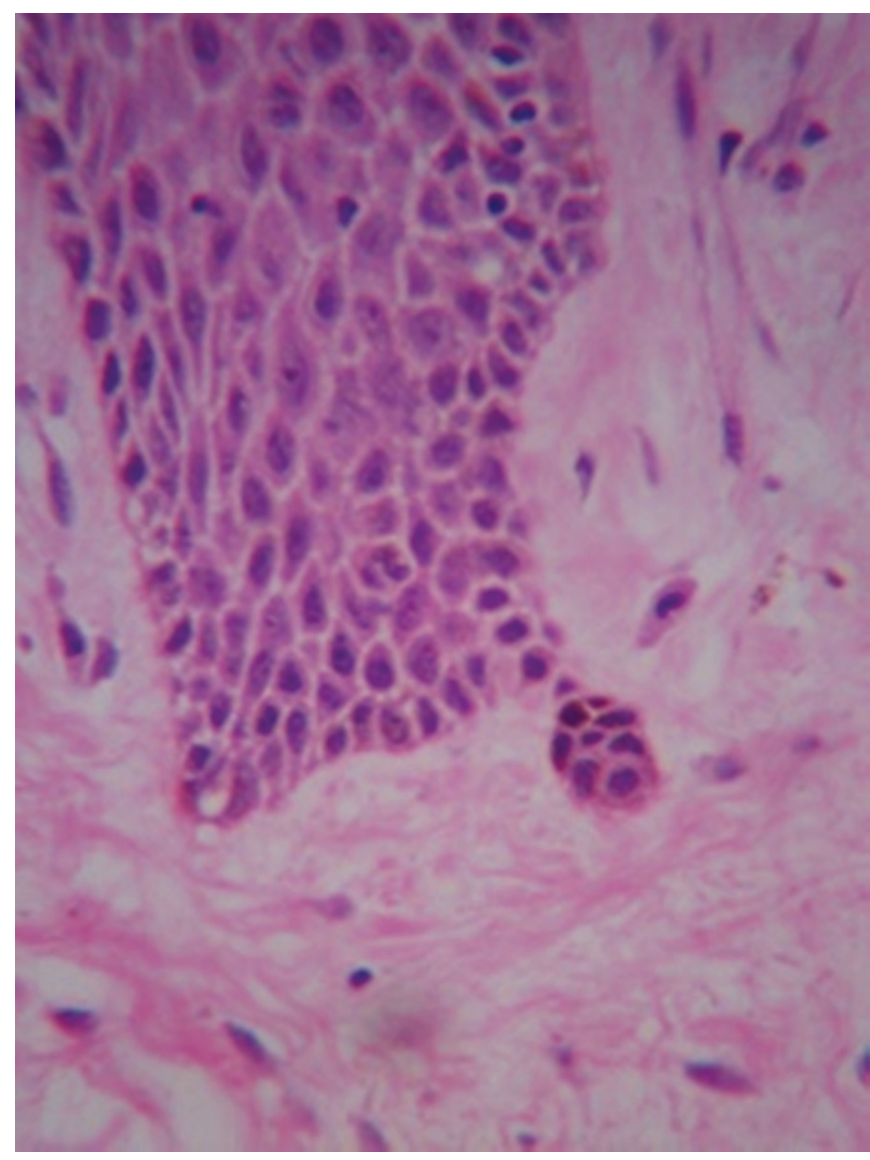

Fig. 7: Histopathological picture showing basal cell budding in DIGO

present as increased gingival size. Such reactive lesions are less commonly present in other intraoral sites, such as cheek, tongue, palate, and floor of the mouth. ${ }^{4}$

Clinically, these reactive lesions often present diagnostic challenges because they mimic various groups of pathologic processes. They are clinically similar but possess distinct histopathological features. ${ }^{4}$
In our study, 50 cases of IGO were considered which showed a female predominance in and most cases were reported in the 3rd and 4th decade in the maxillary anterior region. Pregnant women and women on contraceptives were excluded. Histopathological features showed predominantly hyperplastic epithelium with irregular rete ridges. Connective tissue stroma was predominantly fibrous with most cases showing moderate-todense lymphoplasmacytic inflammatory infiltrate and vascularity. Most cases showed mild fibrosis and hyalinization. There were a few studies reported in the literature on histopathological parameters with respect to gingival overgrowths.

The age prevalence and female predominance seen in our study was similar to the study by Sumarta and Kamadjaja ${ }^{6}$ and Al-Rawi ${ }^{7}$ who studied the prevalence of localized reactive hyperplasic lesions of the gingiva considering the age, sex, site, and the clinical presentation of the lesions and observed that fibrous hyperplasia on the gingiva had similar age, gender, and site preponderance as seen in our study. ${ }^{7}$

The results of our study, however, differed from the study by Ramu and Rodrigues who in their clinico-pathological study concluded that inflammatory hyperplastic gingival lesions occurred in the anterior mandibular region in the 3rd-4th decade more predominantly in males. ${ }^{8}$

DIGO is a widely recognized, unwanted sequela associated with a broad variety of drugs, in which the drugs modify the gingiva. Although the pharmacological effect of drugs is different and directed towards various primary target tissues, all of them seem to act similarly on secondary target tissue, i.e., gingival connective tissue, presenting a common clinical and histopathological feature. Drugs associated with gingival overgrowth can be categorized broadly into three major groups according to their therapeutic actions namely,

1. Anticonvulsants,

2. Immunosuppressants, and

3. Calcium channel blockers. ${ }^{9}$

There is wide variation in the literature regarding the prevalence of drug-induced gingival hyperplasia. For phenytoin, the figure of $50 \%$ is quoted, whereas for cyclosporine and calcium channel blockers (CCBs) a much lower prevalence of 30 and $10 \%$, respectively is reported. Among CCBs, the prevalence of gingival hyperplasia is reported to be maximum for nifedipine (30-50\%) treatment as compared to other CCBs. Reported prevalence of GO after cyclosporine therapy varies between 8 and $81 \% .{ }^{9}$ Drug-induced GO usually occurs within the first three months of starting drug therapy and begins as an enlargement of the interdental papilla. So far, the etiology of DIGO is not fully understood but is clearly multifactorial. ${ }^{10}$

Risk factors for DIGO include age, sex, drug variables, dosage and combination, and genetic factors. ${ }^{9}$

In our study, 23 cases of DIGO were considered, which showed a female predominance with most of the cases reported in the 5 th and 6 th decade in the maxillary posterior region. Predominant cases of DIGO in our study were associated with phenytoin (9 cases) followed by amlodipine ( 7 cases), nifedipine ( 5 cases), and cyclosporine ( 1 case). Clinically, the lesions were pink, firm in consistency with irregular gingival contour. Histopathological features revealed predominantly hyperplastic epithelium with irregular rete ridges. Connective tissue stroma was predominantly fibrous with most cases showing mild-tomoderate lymphoplasmacytic inflammatory infiltrate and vascularity. Most cases showed moderate fibrosis and hyalinization. 
Marshall and Bartold showed that the incidence and the severity of drug-induced gingival overgrowth is greatest on the labial aspects of anterior teeth in contrary to our study, which showed predominance in the maxillary posterior region. ${ }^{11}$

Study by Kantarci et al. ${ }^{12}$ and Lin et al. ${ }^{13}$ showed similar histopathological features of DIGO as those observed in our study along with enlarged rete ridges and discontinuities in the basement membrane of phenytoin-induced DIGO, suggesting that the fibrotic transformation is accompanied by increased extensions of epithelia into the connective tissue..$^{12,13}$

Sume et al. (2010) studied the histopathology of DIGO and reported that the elongated rete ridges in gingival overgrowth may result from an increased level of epithelial plasticity, resulting in mesenchymal characteristics that can be considered to be a phenotypic transition, known as "epithelial-mesenchymal transition". An ultrastructural study demonstrated that increase in the gingival tissue volume is primarily due to a connective tissue response rather than an epithelial layer involvement. ${ }^{14}$

Hereditary gingival overgrowth (HGO) is a rare condition that can occur as an isolated disease or as a part of a syndrome or chromosomal abnormality. Genetic characteristics of HGO have provided novel clues about the potential mechanism of pathogenesis. Histological and cell culture studies have uncovered some of the molecular and cellular changes associated with HGO. However, the pathogenesis of the disease is still largely unknown. ${ }^{15}$

In our study, 17 cases of HGO were considered, which showed a female predominance unlike the study by Xiao et al. (2001), Ye et al. (2005), which showed that both males and females are equally affected by HGO. All the cases of HGO in our study were non-syndromic.

In our study, most of the cases were reported in the 5th and 6th decade in the maxillary anterior region. Clinically, the lesions were pink, firm in consistency with irregular contours. Histopathological features showed predominantly hyperplastic epithelium with irregular rete ridges. Connective tissue stroma was predominantly fibrous with most cases showing mild lymphoplasmacytic inflammatory infiltrate and vascularity. Most cases showed moderate-to-extensive fibrosis and hyalinization, similar to most of the other studies conducted.

In a study by Meng et al. (2008), it was explained that keratinocytes have an important role in the HGO pathogenesis by inducing extracellular matrix (ECM) accumulation by fibroblasts and suggested that keratinocyte-fibroblast interactions contribute to the pathogenesis of $\mathrm{HGO}$, thereby suggesting a possible role of epithelial-mesenchymal interaction in the pathogenesis of $\mathrm{HGO}^{16,17}$

\section{Conclusion}

Gingival overgrowth (GO), in addition to being disfiguring, also leads to poor oral hygiene and debilitated oral functions in individuals. Hence, emphasizing early and appropriate treatment is necessary. Also, dynamics of gingival overgrowth studied by morphology seem likely to further initiate immunological and genetic studies and result in a greater understanding of the biological mechanisms pertaining to it.

This histopathological study lays down the foundation of basic concepts of GO. To conclude, the present study evaluated all three groups of GO to check for their clinical characteristics and histopathological differences, which may help in understanding the aspects of gingival ECM metabolism and its varied response to different stimulus.

\section{References}

1. Banita M, Pisoschi C, Fusaru AM, et al. Myofibroblast involvement in collagen synthesis in gingival overgrowth. Ann Romanian Soc Cell Biol 2009;15(1):129-135.

2. Takei N, Carranza K. Carranza's Clinical Periodontology, 10th edition. Saunders; 2006.

3. Verma PK, Srivastava R, Baranwal HC, et al. "Pyogenic granulomahyperplastic lesion of the gingiva: case reports". Open Dent J 2012;6:153-156. DOI: 10.2174/1874210601206010153.

4. Prasanna J, Sehrawat S. Fibroepithelial hyperplasia: rare, selflimiting condition -two case reports. J Adv Oral Res October 2011;2(3):63-70. DOI: $10.1177 / 2229411220110324$.

5. Damasceno LS, Gonçalves LS, Silva EC, et al. Stromal myofibroblasts in focal reactive overgrowths of the gingiva. Braz Oral Res 2012;26(4):373-377. DOI: 10.1590/s1806-83242012005000012.

6. Sumarta NPM, Kamadjaja DB. Fibrous epulis associated with impacted lower right third molar. Dent J (Maj Ked Gigi) 2009;42(4):172-174. DOI: 10.20473/j.djmkg.v42.i4.p172-174.

7. Al-Rawi NH. Localized reactive lesions of gingiva: a clinicopathological study of 638 lesions from Iraq. Internet J Dent Sci 2009;7:1.

8. Ramu S, Rodrigues C. Reactive hyperplastic lesions of gingiva: a retrospective study of 260 cases. World J Dent 2012;3(2):126-130. DOI: $10.5005 /$ jp-journals-10015-1142.

9. Dongari-Bagtzoglou A. Drug-associated gingival enlargement. J Periodontol 2004;75(10):1424-1431. DOI: 10.1902/jop.2004.75.10.1424.

10. Kumar A, Kumar V, Singh JD. Drug induced gingival hyperplasia: an updated review. Int J Pharmacol Toxicol Sci 2011;1:34-42.

11. Marshall RI, Bartold PM. A clinical review of drug-induced gingival overgrowths. Aust Dent J 1999;44(4):219-232. DOI: 10.1111/j.18347819.1999.tb00224.x.

12. Kantarci A, Nseir Z, Kim YS, et al. Loss of basement membrane integrity in human gingival overgrowth. J Dent Res 2011;90(7):887-893. DOI: 10.1177/0022034511404703.

13. Lin K, Guilhoto LMFF, Yacubian EMT. Drug induced gingival enlargement-Part II. Antiepileptic drugs: not only phenytoin is involved. J Epilepsy Clin Neurophysiol 2007;13(2):83-88. DOI: 10.1590/ S1676-26492007000200009.

14. Sume SS, Kantarci A, Lee A, et al. Epithelial to mesenchymal transition in gingival overgrowth. Am J Pathol 2010;177(1):208-218. DOI: 10.2353/ajpath.2010.090952.

15. Häkkinen L, Csiszar A. Hereditary gingival fibromatosis: characteristics and novel putative pathogenic mechanisms. J Dent Res 2007;86(1):25-34. DOI: 10.1177/154405910708600104.

16. Koyuncu BÖ, Çetingül E, Kandemir Ş, et al. Hereditary gingival fibromatosis: a case report. clinical Dentistry And Research 2012;36:54-58.

17. Nayak PA, Nayak UA, Khandelwal V, et al. Idiopathic gingival fibromatosis. Int J Clin Pediatr Dent 2011;4(1):77-81. DOI: 10.5005/ jp-journals-10005-1086. 